\title{
Feature Extraction for Topological Mine Maps
}

\author{
David Silver, Dave Ferguson, Aaron Morris, and Scott Thayer \\ Robotics Institute \\ Carnegie Mellon University \\ Pittsburgh, PA, USA
}

\begin{abstract}
We present a robust method for detecting and recognizing topological features in underground mines. Our method involves performing Delaunay triangulations on range scans to extract points of interest, such as intersecting corridors. By combining these interest points into a topological map, we have a valuable tool for navigation and localization in large scale, highly cyclic environments. We present results from a research coal mine near Pittsburgh, PA.
\end{abstract}

\section{INTRODUCTION}

The navigation and mapping of underground mines presents both an opportunity and a significant challenge for the field of robotics. In the U.S. alone, tens of thousands of abandoned mines threaten nearby surface structures, ecosystems, and ongoing mining operations [1]. Proactive measures can be taken to prevent these threats from manifesting into disastrous situations; however, prevention relies upon knowledge of mine layout and condition. Mine maps are the primary source of such information. In the case of abandoned mines, these maps, when they exist, are often inaccurate if at all legible.

Robots are sensible tools for mapping mines since they can potentially penetrate areas too small or too dangerous for people to enter. Autonomous mine navigation for mobile robots, however, is a tremendous challenge as underground mines are highly cyclic, rough environments. Generating consistent large-scale maps in real-time for the purpose of robot navigation is a difficult process [2].

In this paper, we present a method for the extraction of topological features in underground mines based on the theory of Voronoi diagrams and Delaunay triangulations [3]. These methods enable robust and consistent detection of corridor intersections for the creation of topological mine maps. This method can also extract unique characteristics of each labeled feature for both localization and highlevel navigation. A full set of unique features can provide global landmarks that can correct accumulated error in the mapping system and assist in loop closure.

We begin by introducing our robot "Groundhog" and provide some motivation for our current work. In Section III we discuss related research in topological mapping. We present our approach in Section IV and results from an experimental mine in Section V. We conclude by proposing a number of extensions to the current system.

\section{The Mine Mapping Project}

Our current research focuses on the problem of autonomous mine mapping. We are interested in both the

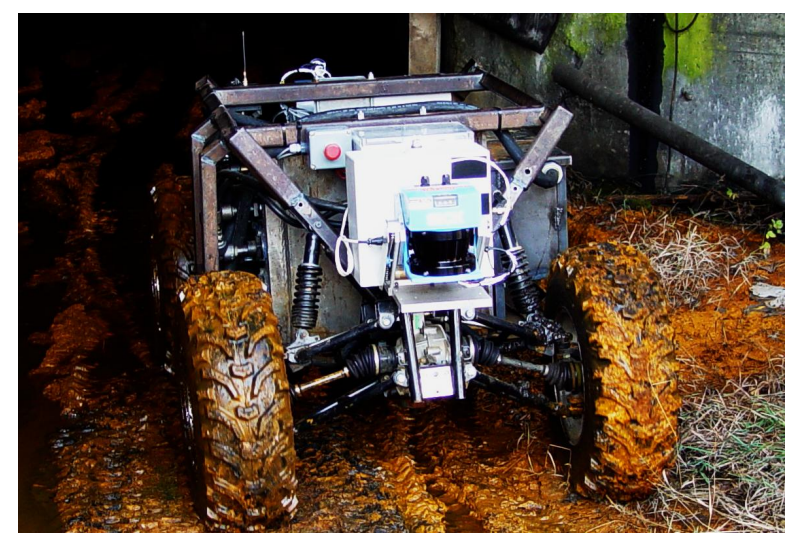

Fig. 1. Groundhog, our current robotic mine mapping platform

creation of systems that can robustly explore mine environments and the development of algorithms that can generate accurate maps of these environments.

Our current robotic system, Groundhog (Figure 1), is a $700 \mathrm{~kg}$ custom-built platform equipped with onboard computing, laser range finders, gas sensors, and a low-light digital video camera. Groundhog is constructed from two reinforced ATV front-ends, and is rugged enough to cope with the difficult terrain inherent in abandoned mines. For hardware details, see [4].

The Groundhog platform has been used extensively in both test mine and abandoned mine environments. Groundhog has accrued in excess of fifty hours of mine navigation including 8 successful experiments in the abandoned Mathies mine outside of Pittsburgh, PA [4]. Over the course of its lifetime, Groundhog has evolved into a system that is highly proficient at traversing mine corridors autonomously, successfully navigating over $2 \mathrm{~km}$ of abandoned mine corridor.

In autonomous operation, Groundhog first pauses to take a 3D scan of its local environment using a scanning laser range finder mounted on the front of the robot. This scan data is then projected onto a $2 \mathrm{D}$ gradient map, which is used to plan local paths. Paths are then executed with the assistance of a 2D scan matcher for position estimation. Upon reaching the terminal point of its path, it repeats the sense, plan, and act cycle. This approach enables Groundhog to consider 3D mine structure for safe traversal of mine corridors.

In its current state, Groundhog is unable to perform general mine exploration. Achieving this capability would require both the detection and unique identification of in- 
tersections for localization and global navigation. In mines, intersection identification and classification are complementary problems. Intersections encompass a variety of geometric structures since mine corridors rarely cross at right angles with well defined corners. These conditions make it difficult to robustly detect intersections; however, the large structural variance also provides ample uniqueness to each intersection such that reliable classification is possible. Therefore, consistent and reliable topological feature detection is a critical step towards general exploration.

\section{TOPOLOGICAL MAPPING}

\section{A. Topological Maps}

Mine environments, which consist almost exclusively of narrow corridors and corridor intersections, are ideally suited for Topological Mapping. A topological map [5] is a graph representation of an agent's environment. Nodes in the graph correspond to distinct locations in the environment (intersections), while edges correspond to a direct path between two such locations (corridors). A major advantage of topological maps over grid or feature based approaches is a huge reduction in both the space and time complexity of the representation and its associated algorithms [6].

Topological maps have proven to be very useful in robotic exploration tasks [7]. Unexplored edges in a topological map correspond to unexplored regions of the robot's environment. If the chosen topology is a roadmap [8], exploring every edge in a topological map will allow the robot to explore every part of the freespace. This can be seen as accomplishing a sensor coverage task.

Implicit in this idea is that the robot has some way of determining node locations from its sensor data, as well as the edges leaving each node. Once on an edge, the robot must be able to follow that edge to the next node. A topology that is detectable from local sensor data is said to be embedded in the robot's environment.

\section{B. Topological SLAM}

Localization with a topological map can be performed by exploiting the nature of the representation. When a robot is at a node of an embedded topology, then in the absence of any other information about the robot's location, it knows it is at one of a finite set of points in the environment (the set of nodes). If a method exists for the robot to distinguish which node from within this set it is presently at, then the robot can determine its location in the environment simply by looking up that node's location in the topological map [9]. Such a localization procedure depends on a previously constructed topological map being available to the robot. It does not depend on knowing the exact location of the node in the environment. This allows the robot to navigate with regard to the topology, without knowing its exact global location.

When the robot explores an unknown environment and preexisting maps are unavailable, it must engage in Simultaneous Localization and Mapping (SLAM) [10]. In this context, a topological map is built while the robot performs its exploration. Given a static environment, node locations remain at fixed points, so returning to a previously visited node provides an opportunity to determine and correct for positioning error. This is referred to as topological SLAM [11].

Performing topological SLAM in an acyclic environment is relatively straightforward. The first node the robot detects is made the root of the topological map. From then on, the robot has a good idea of whether it is visiting a new node or a previously explored node, just by keeping track of whether the current edge has already been traversed.

Unfortunately, most environments, and especially mines [12], contain cycles. Thus, every time the robot finishes exploring a new edge, it must consider the possibility that the next node has been previously visited and already exists in its topological map. This is known as "closing the loop". Topological SLAM in such an environment requires a robust method for performing this type of reasoning at each stage of exploration.

Autonomous loop closure with topological maps has recently been demonstrated by Lisien et al [13]. When arriving at a node which may correspond to a node already in the topological map, they use embedded properties of each node to reduce the set of possible correspondences, then restrict this set further by the use of engineered landmarks along each edge.

In the following section, we describe our method of topological feature extraction. We begin with some background on Voronoi diagrams and Delaunay triangulations. We go on to describe how these ideas can be used to extract interesting topological features from mine environments, and how these features can be combined into topological maps for navigation and localization.

\section{Topological Feature Extraction}

\section{A. Voronoi Diagrams and Delaunay Triangulations}

Consider a set of points $P$ in the plane. For every point $p_{i}$ in $P$, the region of the plane that is closer to $p_{i}$ than to any other point in $P$ is the Voronoi region associated with $p_{i}$, denoted $V_{i}$. Every point in the plane belongs to a Voronoi region. The boundary between two adjacent regions $V_{i}$ and $V_{j}$ is the set of points that are equidistant to $p_{i}$ and $p_{j}$, and are closer to these two points than any other point in $P$. This boundary is a Voronoi edge, denoted $E_{i j}$. Every Voronoi edge either extends to infinity, or terminates at an intersection with two other Voronoi Edges. Such an intersection occurs at a point in the plane that is equidistant to 3 points $p_{i}, p_{j}$ and $p_{k}$ in $P$, and is closer to these three points than any other point in $P$. A point of three way equidistance is called a Voronoi node, denoted $N_{i j k}$. The set of Voronoi nodes and Voronoi edges of a particular point set is called the Voronoi diagram.

Different types of Voronoi diagrams can be produced by changing the definition of distance. When the distance metric used is the minimum distance to a single object in the plane, rather than to a single point, the resulting structure is called the generalized Voronoi diagram (GVD)[14]. The GVD is an embedded roadmap that can be traced 

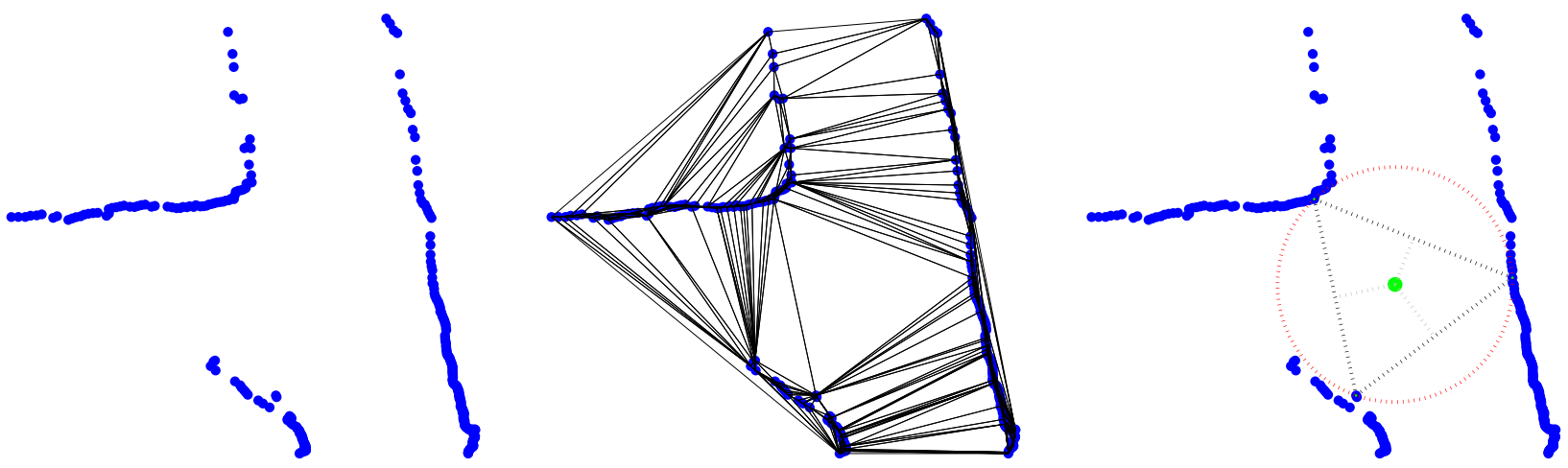

Fig. 2. Extracting Features. On the left are the 2D points generated from a single range scan. In the center are the Delaunay triangles returned from this set of points. On the right are the extracted features after thresholding the triangles and computing the center points and radii for those that remain.

from purely local sensory input, making it well suited for topological mapping [11], [13].

The Delaunay Triangulation of $P$ is the set of triangles $T$ such that for every Voronoi node $N_{i j k}$, there is a triangle in $T$ that has $p_{i}, p_{j}$, and $p_{k}$ as its corners. This duality is symmetric: every triangle $T_{i j k}$ corresponds to a Voronoi node $N_{i j k}$ and the sides of the triangle are bisected by Voronoi edges $E_{i j}, E_{j k}$, and $E_{i k}$. From this duality, it can be shown that the Delaunay triangulation is a proper triangulation of the set $P$ in the plane. Another result of this duality is that the circle centered at $N_{i j k}$ and passing through the points $p_{i}, p_{j}$, and $p_{k}$ (the circumcircle of $T_{i j k}$ ) contains no other points in $P$. For a detailed discussion of Voronoi diagrams and Delaunay triangulations, see [3].

\section{B. Topological Features}

The Delaunay triangulation has many properties that lend to robust feature extraction from a planar point set [15], [16]. The Delaunay triangulation is not affected by rotations and translations applied to a point set; the same points in the set form Delaunay triangles at their new locations. Also, the Delaunay triangulation is robust with respect to variations in point locations that are small compared to the scale of the triangle. Finally, changes to a small region of the point set do not propagate over the entire triangulation, they have only local influence.

These properties motivate the application of Delaunay triangulations to planar point sets created from laser range scans. The idea is that a section of the environment that is scanned from different robot configurations should have approximately the same Delaunay triangulation once the displacement of the robot is taken into account. The differences in the two triangulations should then be solely due to sensor noise.

The variation in the triangulation due to noise will depend on the size of the triangle in question. The shorter the side of a triangle, the more likely that side is to change endpoints due to noise. Conversely, the longer the side of a triangle, the more stable it is to noise. Triangles that have three long sides are therefore robust features of the environment.

Our feature extraction approach exploits these characteristics as follows. First, the Delaunay triangulation is computed from a laser range scan. Next, the sides of each resulting triangle are tested against a distance threshold $D_{\text {min }}$. This threshold is set equal to the minimum corridor width the robot is able to traverse. If any side of a triangle does not pass this threshold, that triangle is eliminated. It has been empirically observed that obtuse Delaunay triangles are not as robust regardless of size, and so these triangles are also removed. The remaining triangles are stable, and their corresponding Voronoi nodes provide robust features. This process is illustrated in Figure 2.

\section{Topological Maps from Topological Features}

To understand why this approach produces robust topological features, it must be viewed from a Voronoi context. As mentioned previously, each side of a Delaunay triangle corresponds to a Voronoi edge $E_{i j}$. Each Voronoi edge bisects the line between the corresponding points $p_{i}$ and $p_{j}$. If the distance between these two points (the length of the side of the triangle) is less than $D_{\min }$, then the robot will be unable to traverse this edge. So our thresholding procedure implicitly eliminates all Voronoi edges that pass too close to the boundary of freespace for the robot to trace. Since a Voronoi node is the mutual endpoint of three Voronoi edges, eliminating Voronoi edges eliminates Voronoi nodes. What is left is the set of Voronoi nodes for which all neighboring edges are traversable by the robot.

This procedure is similar to that used by Mahkovic and Slivnik in [17] to construct what they term the generalized local Voronoi diagram (GLVD). The GLVD is a local approximation of the GVD built by clustering points together into objects and computing the distance to these objects. A key difference is that the clustering used by our approach is not explicitly performed, but rather is inferred from the Delaunay triangulation. Also, our clustering threshold is defined not in terms of the local environment, but by the physical dimensions of the robot. In this sense, our approach can be seen as approximating the GVD in the robot's configuration space.

Since the GVD is an embedded roadmap, a topological map may be constructed from the extracted features that will allow for coverage of the entire space. However, not all of the extracted topological features may correspond to corridor intersections; any sufficiently large concavity 

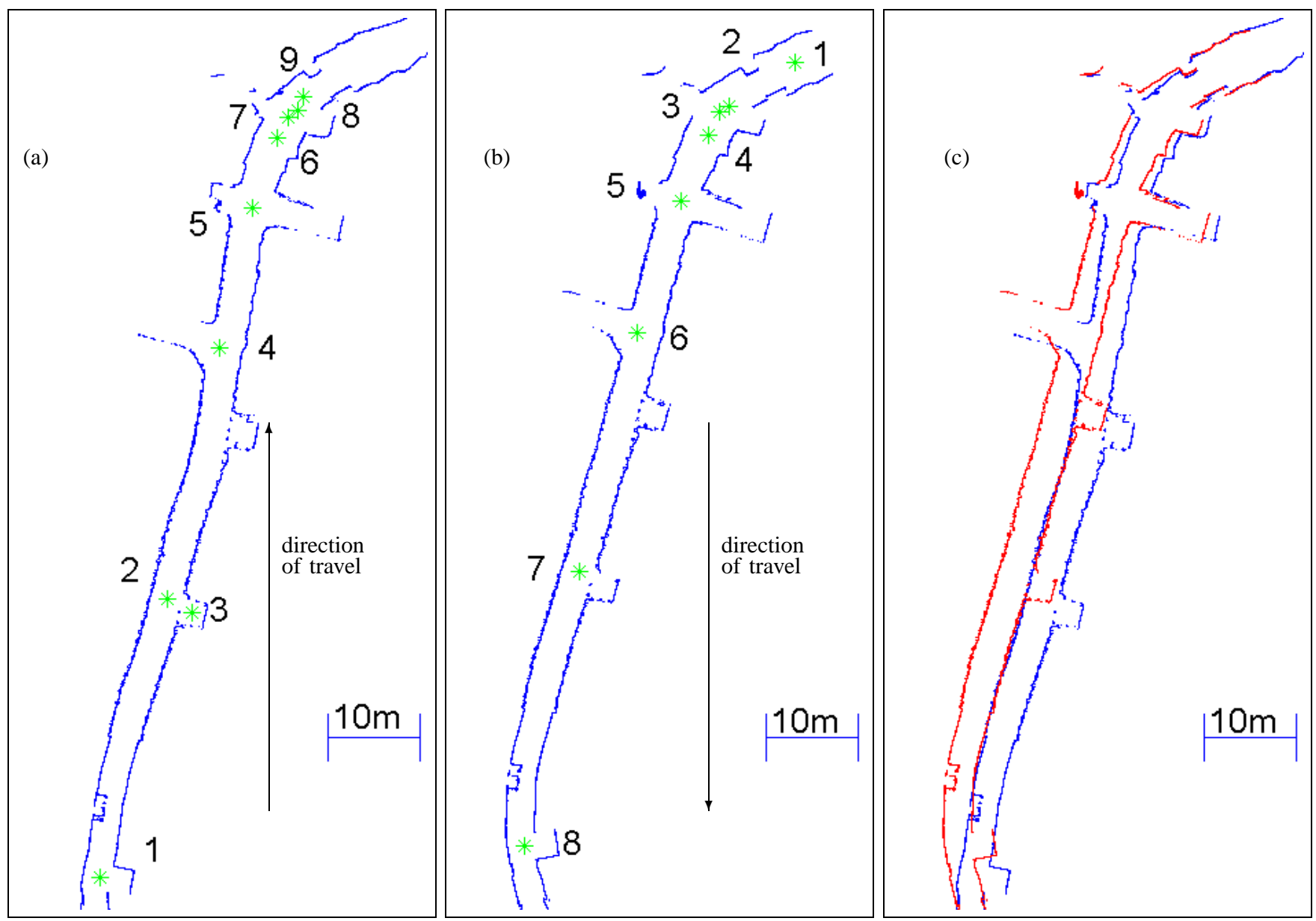

Fig. 3. Example of the feature extraction and topological map construction. (a) The map created on the traverse into the mine. (b) The map created on the way out. (c) The two maps overlaid onto one another. Notice the features in each map are in close correspondence even though the maps themselves are not.

will satisfy our extraction algorithm. To determine whether a topological feature corresponds to an intersection, the Voronoi edges leaving a node are checked to determine whether they will terminate near an obstacle within the robot's sensor range. If any edges leaving the node do terminate in such a way, than that edge is not worth exploring (the robot can already see the end of it) and so the node is not an intersection of sufficiently long corridors. This is equivalent to only keeping features that correspond to nodes of the reduced generalized Voronoi diagram [18] of the local environment. These are termed 'strong' nodes; nodes with boundary edges are termed 'weak' since they are more likely to appear and disappear due to sensor noise. A topological map may then be built from only strong nodes that provides the same coverage guarantee as the full GVD. The remaining topological features may then be stored purely for feature matching (Section IV-D), or alternatively may be used in a traditional landmark based SLAM architecture [19].

Since Voronoi nodes by definition are of degree three, special care must be taken with intersections of more than three corridors. In that case, a group of topological features will be detected within a very small area. In the absence of noise, each of the triangles corresponding to these features will share an edge with some other triangle in the group. Therefore, these intersections can be correctly classified by checking pairs of triangles to determine if they share a side, within some noise tolerance.

\section{Feature Matching}

Another nice property of these features is that they have a number of characteristics that can be used to detect correspondences between observed features. Along with the position of the feature, these include: the degree of the associated node, the radius of the circumcircle of the associated triangle, and the relative angles of the edges leaving the node. In [11], it was stated that relative edge angles are not a stable characteristic of a GVD node. This was empirically observed in [13], as well as in our own experiments. Therefore, we use only the radius and degree of the node. These attributes can be combined with local scan information to produce feature correspondences even without reliable position estimation, as described in the next section. 

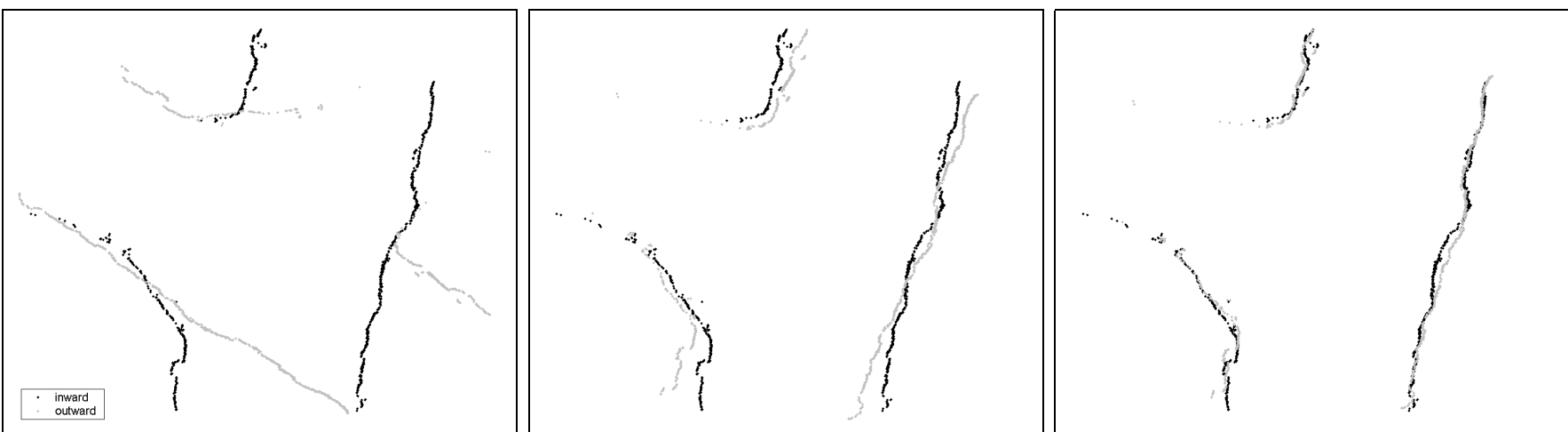

Fig. 4. Matching Features. On the left are two local range maps, centered by their feature points, overlaid on one another. In the center is the minimum error match using pure rotation in increments of 10 degrees. On the right is the final ICP minimum error match.

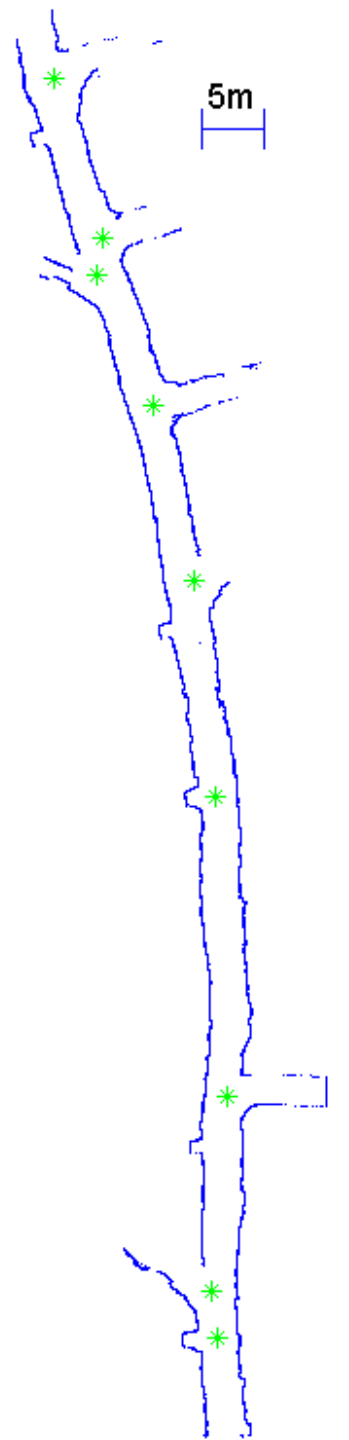

Fig. 5. Topological features extracted from a second mine corridor

\section{RESULTS}

In order to test our topological feature detection algorithm, a set of experimental runs was performed in a research coal mine near Pittsburgh. For each run, features were first extracted on a per scan basis. Features that appeared in several consecutive scans were then recorded. The first experiment was conducted inside a feature-rich length of corridor to test the reliability of feature detection. The second experiment was conducted in a separate stretch of mine corridor for testing the consistency of features taken from different vantages. Each corridor used for testing exhibited many of the structural and environmental characteristics found in underground mines: intersections, minor excavations, mine carts and other equipment. Groundhog autonomously traversed these corridors and produced the maps seen throughout this section.

In the first experiment, Groundhog traversed a relatively straight corridor that contained several intersections and excavated sections. Figure 5 displays the map and topological interest points detected along the 100 meter stretch of mine corridor. In total, 9 features met the criteria for interest point selection. Of these 9 features, 5 features corresponded to the 5 intersections within this section of corridor. The remaining features were pockets of excavated coal or other concavities. Altogether, the feature detector correctly identified all corridor intersections as topological features, thus demonstrating the reliability of this approach.

For the second experiment, Groundhog traversed another 100 meter stretch of mine corridor in two directions: inwards and outwards. These two traverses examined the same section of mine from different vantages to test preliminary correspondences between feature characteristics. Figure 3(a) shows the map created along the inward traverse with the extracted feature points. Figure 3(b) shows the map and features generated on the outward traverse, after the robot autonomously reversed its direction at the end of its inwards run. In total, 10 unique features were identified where 7 of these feature appeared in both traverses. The 3 that did not appear in both traverses occurred as a result of occlusions made visible and vice versa when the vantage was flipped. These non-consistent features were found to be weak nodes, which do not effect the overall topology.

As shown by the overlay of these maps in Figure 3(c), 

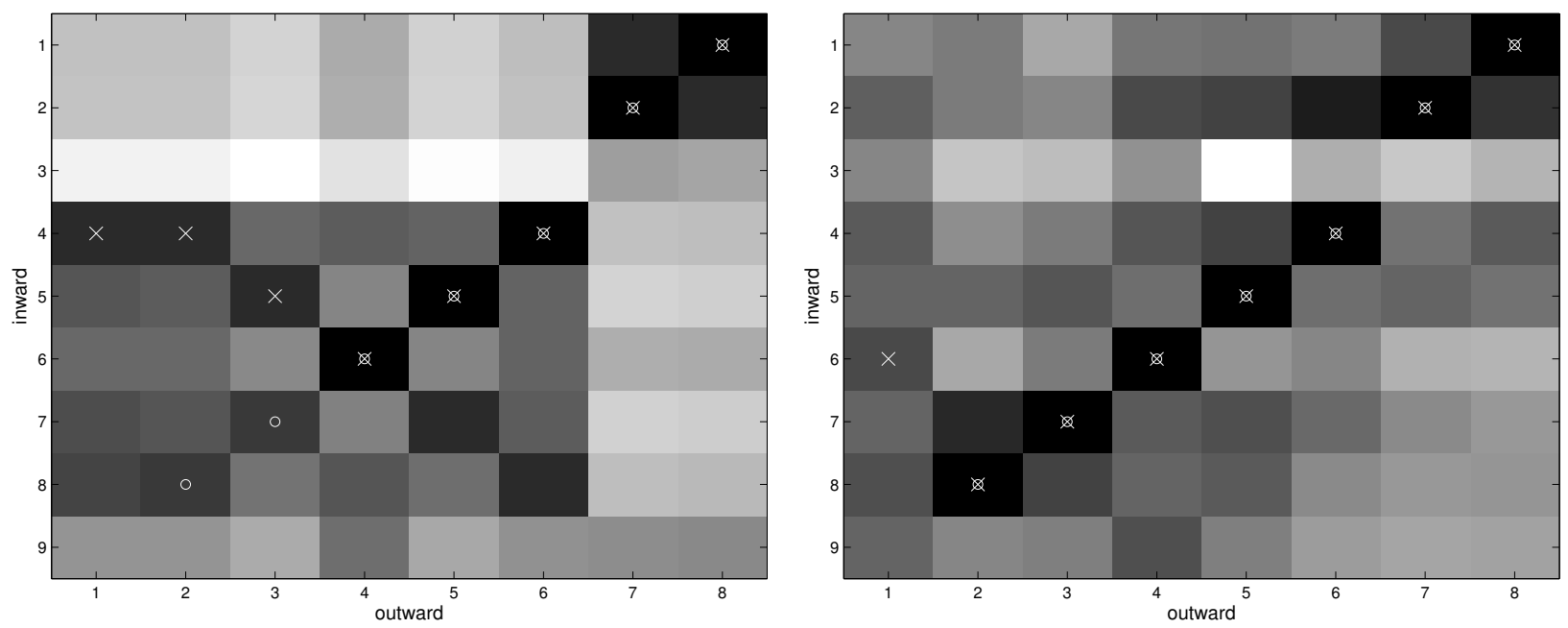

Fig. 6. The pairwise matching error between the features recorded during the inwards and outwards runs. On the left is the difference between the radii of each pair of features, on the right is the minimum error achieved by performing ICP with the two feature point clouds. A darker cell indicates less error. The true correspondences are labeled with white circles. The minimum error correspondences(from the outward to the inward traverse) are labeled with white 'X's

no position or feature information was utilized between experiments. Both the inwards and outwards traverses were treated as independent runs. Thus, in both runs the 2 intersections in this corridor were correctly identified along with 5 other topological areas of interest. Such consistency across multiple independent runs from different vantages demonstrates the potential for reliable topological map generation.

In addition to extracting topological features from both traversals in the second experiment, preliminary feature matching between these sets was performed using two criteria. The first criteria correlated features by comparing the radii of the feature circumcircle between the inwards and outwards run. Figure 6(a) shows the correspondence among these features and demonstrates that radii alone are reasonable indicators of feature correspondences. While radii comparison is not a completely accurate method of comparison, it can be used to reduce the set of possible correspondences for better, more computationally expensive correspondence methods.

The second criteria compared local range maps acquired around each feature for detecting correspondences. In this comparison, each selected feature from both runs had an associated set of 2-D range scans that best represented the local geometry of the interest point. Using the Iterative Closest Point (ICP) [20] algorithm, each feature was compared with every other identified feature. To ensure completeness of this comparison, both sets of scans were translated so that the feature point was at the origin. Next, one scan was held fixed while the other was rotated in 10 degree increments. For each rotation, the ICP error was calculated. After rotating a full 360 degrees, the minimum error rotation across all angles was used to seed ICP. This rough scan of the entire space of rotations is necessary to ensure proper convergence of ICP; Voronoi edge angles were found to contain too much noise to provide a reliable alignment.
After computing the seed displacement, ICP was run until convergence. The final ICP error was considered as the final correspondence value between the two sets of scans. Figure 4 shows the pairwise matching process. Figure 6(b) shows the final error returned by ICP for each pair of features. As can be seen from the figure, the minimum error correspondence is the correct correspondence in each case.

\section{DISCUSSION}

In summary, our results have demonstrated the concepts of reliable topological feature detection in coal mine environments. In both experiments, every intersection was identified as a feature. This kind of detection consistency is required since intersections will ultimately form the nodes in a topological representation of the mine. This topological representation will also form the basis of many autonomous processes such as navigation, localization, and general purpose exploration.

In addition to intersection detection, our feature identification method was shown to be beneficial in the correlation of interest points, even from varying perspectives and without any regard to approximate feature position. With information about the radii corresponding to each feature, several un-correlated features can be eliminated with minimal computational overhead. This correlation can be refined further using ICP on the $2 \mathrm{D}$ geometric data surrounding the points of interest. Although this refinement may not always guarantee an absolute correlation between two features, it will significantly reduce the size of the candidate set.

While feature identification is a necessary step towards reliable autonomy in coal mines, it is only the base component in a number of processes that will push Groundhog and the mine mapping project towards the goal of general purpose mine exploration. With robust feature detection and intersection classification, a reliable topological map 
of these mine spaces can be constructed. As discussed previously, these maps are excellent geometric representations of mine environments with little computational or memory overhead for storage or processing. Such maps are ideal for global navigation.

In addition to feature detection and classification, a $3 \mathrm{D}$ correlation process is required to accurately build the topological map. Such a process will enable loop closure in these topological structures as well as localization of the robot to a specific node in the map with certainty. Taking advantage of the feature-rich, and unique nature of these mine intersections, the challenge of loop closure is greatly simplified. As already shown, with 2D information our method was capable of correctly establishing feature correspondences from different vantages. While we know absolute correlation may not always occur, using these 2D methods to efficiently reduce the search space will allow more complex $3 \mathrm{D}$ registration techniques to be feasible in real time.

While the future challenges and possible solutions are well known, much remains to be done. We are currently implementing extensions of the feature extraction and localization algorithm on Groundhog that would enable control decisions to be influenced by the uncertainty associated with its position within its topological map (e.g. when closing a large loop). We are also incorporating the $3 \mathrm{D}$ scan information into into the process for more accurate classification and correlation. Finally, we are exploring the possibility of incorporating the extracted features not used as nodes into a traditional landmark based SLAM framework [19].

\section{CONCLUSION}

We have presented a robust method for detecting and recognizing topological features in underground mines. To detect features, our approach involves performing Delaunay triangulations on range scans and restricting attention to the subset of resulting triangles that are of a sufficient size and persist over a number of consecutive scans. These features correspond to nodes of the generalized Voronoi diagram. Corridor intersections form a subset of these features. Feature matching is performed by comparing pairs of features in terms of their estimated positions, radii, and local point clouds. The resulting system can be used to perform localization and high-level navigation in mine environments.

\section{ACKNOWLEDGMENTS}

Chris Baker, Charlie Reverte, Zach Omohundro, Chuck Whittaker and Red Whittaker for their tireless efforts that made this work possible.

The Carnegie Mellon HSLAM group, for continued inspiration and feedback.

The National Institute of Occupational Health and Safety, Mine Safety and Health Administration, Pennsylvania Department of Environmental Protection, and the various people in the mining industry who provided guidance and support for this work.
Workhorse Technologies, LLC, for technical and operational support in all aspects of the project.

Senator Arlen Specter for his continued support of this work.

\section{REFERENCES}

[1] J. Belwood and R. Waugh, "Bats and mines: Abandoned does not always mean empty," Bats, vol. 9, no. 3, 1991.

[2] D. Ferguson, A. Morris, D. Hähnel, C. Baker, Z. Omohundro, C. Reverte, S. Thayer, C. Whittaker, W. Whittaker, W. Burgard, and S. Thrun, "An Autonomous Robotic System for Mapping Abandoned Mines," in Proceedings of the Conference on Neural Information Processing Systems (NIPS). MIT Press, 2003.

[3] F. P. Preparata and M. I. Shamos, Computational Geometry. Springer-Verlag, 1985.

[4] C. Baker, A. Morris, D. Ferguson, S. Thayer, C. Whittaker, Z. Omohundro, C. Reverte, W. Whittaker, D. Hähnel, and S. Thrun, "A Campaign in Autonomous Mine Mapping," in Proceedings of the IEEE International Conference on Robotics and Automation (ICRA), New Orleans, LA, 2004.

[5] B. Kuipers and Y. Byan, "A robot exploration and mapping strategy based on a semantic hierarchy of spatial representations," J. Robot. Auton. Syst., vol. 8, pp. 47-63, 1991.

[6] T. H. Cormen, C. E. Leiserson, R. L. Rivest, and C. Stein, Introduction to Algorithms, Second Edition. MIT Press, 2001.

[7] G. Dudek, M. Jenkin, E. Milios, and D. Wilkes, "Robotic exploration as graph construction," Transactions on Robotics and Automation, vol. 7, no. 6, pp. 859-865, December 1991.

[8] J. Canny, "Constructing roadmaps of semi-algebraic sets I: Completeness," Artif. Intell., vol. 37, pp. 203-222, 1988.

[9] G. Dudek, M. Jenkin, E. Milios, and D. Wilkes, "Map validation and robot self-location in a graph-like world," Robotics and Autonomous Systems, 1998.

[10] J. Leonard and H. Durrant-Whyte, "Simultaneous Map Building and Localization for an Autonomous Mobile Robot," in IEEE/RSJ International Workshop on Intelligent Robots and Systems, May 1991, pp. 1442-1447.

[11] H. Choset and K. Nagatani, "Topological simultaneous localization and mapping (slam): towards exact localization without explicit localization," IEEE Transactions on Robotics and Automation, vol. 17, no. 2, pp. 125-137, Apr. 2001.

[12] A. B. Cummins and I. A. Given, Eds., SME Mining Engineering Handbook. Port City Press, 1973, vol. 1.

[13] B. Lisien, D. Morales, D. Silver, G. Kantor, I. Rekleitis, and H. Choset, "Hierarchical simultaneous localization and mapping," in IEEE/RSJ Int. Conference on Intelligent Robots and Systems, vol. 1 , Oct. 2003 , pp. $448-453$.

[14] H. Choset and J. Burdick, "Sensor based planning, part II: Incremental construction of the generalized voronoi graph," in Proc. of IEEE Conference on Robotics and Automation. Nagoya, Japan: IEEE Press, May 1995, pp. 1643 - 1648.

[15] Y. Xiao and H. Yan, "Facial feature location with delaunay triangulation/voronoi diagram calculation," in Proceedings of the PanSydney area workshop on visual informtaion processing conference on Visual information processing 2001. Australian Computer Society, Inc., 2001, pp. 103-108.

[16] Y. Tao and W. Grosky, "Delaunay triangulation for image object indexing: A novel method for shape representation," in Proceedings of the Seventh SPIE Symposium on Storage and Retreival for Image and Video Databases, 1999, pp. 631-642.

[17] R. Mahkovic and T. Slivnik, "Constructing the generalized local Voronoi diagram from laser range scanner data," IEEE Transactions on Systems, Man, and Cybernetics, Part A, vol. 30, pp. 710-719, 2000 .

[18] K. Nagatani and H. Choset, "Toward robust sensor based exploration by constructing reduced generalized voronoi graph," in IEEE/RSJ Int. Conf. on Intelligent Robots and Systems, 1999.

[19] R. Smith, M. Self, and P. Cheeseman, "Estimating uncertain spatial relationships in robotics," Autonomous Robot Vehicles, pp. 167-193, 1990.

[20] P. J. Besl and N. D. McKay, "A method for registration of 3-d shapes," IEEE Trans. Pattern Anal. Mach. Intell., vol. 14, no. 2, pp. 239-256, 1992. 